Nur Hashima Abdul Rashid, MBBS'

Suria Hayati Md Pauzi, MD²

Geok Chin Tan, MBBS, MPath ${ }^{2}$

Salina Husain, MBBS, MS (ORL-HNS)

Mohd Razif Mohammad Yunus, MBBS, MS (ORL-HNS)'

Balwant Singh Gendeh, MBBS, MS (ORL-HNS)'

'Department of Otorhinolaryngology

Head and Neck Surgery

Universiti Kebangsaan Malaysia Medical Center

Cheras, Kuala Lumpur, Malaysia

2Department of Pathology

Universiti Kebangsaan Malaysia Medical Centre

Jalan Yaacob Latif, 56000 Cheras

Kuala Lumpur, Malaysia
Correspondence: Dr Nur Hashima Abdul Rashid

Department of Otorhinolaryngology and Head and Neck Surgery

Universiti Kebangsaan Malaysia Medical Centre

Jalan Yaacob Latif, 56000

Cheras, Kuala Lumpur

Malaysia

Phone: 603-91456054/6045

Fax:603-91737840

E-mail:nurhashima@gmail.com

Reprints will not be available from the author.

The authors declared that this represents original material that is not being considered for publication or has not been published or accepted for publication elsewhere, in full or in part, in print or electronic media; that the manuscript has been read and approved by all the authors, that the requirements for authorship have been met by each author, and that each author believes that the manuscript represents honest work.

Disclosures: The authors signed disclosures that there are no financial or other (including personal) relationships, intellectual passion, political or religious beliefs, and institutional affiliations that might lead to a conflict of interest.

\section{Nonkeratinizing Carcinoma of the Sinonasal Tract: A Diagnosis of Confusing Nomenclature}

\begin{abstract}
Objective: To describe a rare case of nonkeratinizing carcinoma of the sinonasal tract and review
\end{abstract} the literature on the nomenclature of its many synonyms.

\section{Methods:}

Design: Case Report

Setting: Tertiary Referral Center

Patient: One

Results: A 45-year-old female presented with a 6-month history of left nasal obstruction associated with epistaxis. Computed tomography revealed a mass expanding the left nasal cavity with the epicenter arising from the anterior ethmoidal air cells. Endoscopic resection of the tumor was carried out but as there was residual tumor, she then underwent endoscopic-assisted medial maxillectomy via a lateral rhinotomy. A subsequent computed tomography scan showed residual tumor adhering to the ipsilateral periorbita. The patient has so far declined intensity modulated radiotherapy that was advised though she is still under regular follow-up.

Conclusion: Nonkeratinizing carcinoma of the sinonasal tract is a rare entity and there are very few reports concerning this type of malignancy. This may be partly due to its many synonyms, such as cylindrical cell carcinoma, Schneiderian carcinoma and transitional cell carcinoma. Nomenclature of this tumor should be standardized to avoid confusion and misdocumentation.

Keywords: nonkeratinizing carcinoma, Schneiderian carcinoma, transitional cell carcinoma, cylindrical cell carcinoma, Ringertz carcinoma, respiratory epithelial carcinoma

Non-keratinizing carcinoma of the sinonasal cavity is a rare entity. There are very few reports concerning this type of malignancy. ${ }^{1,2,3}$ This may be partly due to the many different terminologies by which it has been referred to, such as cylindrical cell carcinoma, Schneiderian carcinoma and transitional cell carcinoma. We present a case of a sinonasal non-keratinizing carcinoma. The nomenclature and cytological aspects of this tumor will be discussed in detail.

Philipp J Otolaryngol Head Neck Surg 2011; 26 (2): 21-24

(c) Philippine Society of Otolaryngology - Head and Neck Surgery, Inc. 


\section{CASE REPORT}

A 45-year-old female schoolteacher of Chinese descent presented to a private hospital with a 6-month history of progressively worsening left nasal obstruction associated with epistaxis. There were no other symptoms in this previously well lady. Clinical examination showed a friable mass occupying the left nasal cavity pushing the nasal septum to the opposite side. A complete head and neck examination did not reveal any evidence of cervical lymphadenopathy. A computed tomography scan of the paranasal sinuses showed a predominantly homogeneously-enhancing mass expanding the left nasal cavity and pushing the nasal septum to the right with inferior compression of the ipsilateral inferior turbinate. The epicenter of the mass appeared to be within the left anterior ethmoidal air cells. There was also thinning and displacement of the ipsilateral lamina papyracea noted but no evidence of subperiosteal or extraconal extension within the left orbit. A partial medial maxillectomy via lateral rhinotomy was aborted due to excessive intraoperative bleeding from the tumor. A month later, the patient was referred to us for further management of the residual tumor. Histopathologic examination revealed a non-keratinizing (transitional cell) carcinoma.

Post-operative computed tomography of the paranasal sinuses showed an enhancing soft tissue mass occupying the left nasal cavity, extending antero-superiorly into the ethmoid and frontal sinuses and encroaching postero-superiorly the sphenoid sinus ostium. The left medial rectus muscle was pushed toward the orbit although the fat plane between mass and the muscle was preserved. There was dehiscence of the anterior and medial walls of the maxillary sinus and lamina papyracea (Figure 1). We proceeded with a biopsy of the tumor in the outpatient clinic which was reported as non-keratinizing (transitional cell) carcinoma.

Endoscopic removal of the tumour was planned and an angiogram performed in view of the previous intraoperative bleeding. The feeding vessel was found to be the left internal maxillary artery (a branch of the sphenopalatine artery) and this was subsequently embolized. Intraoperatively there was dehiscence of the medial wall of the maxillary sinus and the ipsilateral lamina papyracea was absent. Ethmoidectomy, sphenoidectomy and frontal sinustomy were performed. The tumor occupying the maxillary, ethmoid, frontal and sphenoid sinuses was removed completely. Tumor adhering to the periorbita was curetted. Despite embolization, bleeding was profuse and the patient was nursed in the intensive care unit with blood and fluid resuscitation and recovery was uneventful.

The patient was asymptomatic during post-operative followup, until ten weeks later when she reported a recurring epistaxis. Endoscopic examination revealed a fleshy mass over the left periorbita.

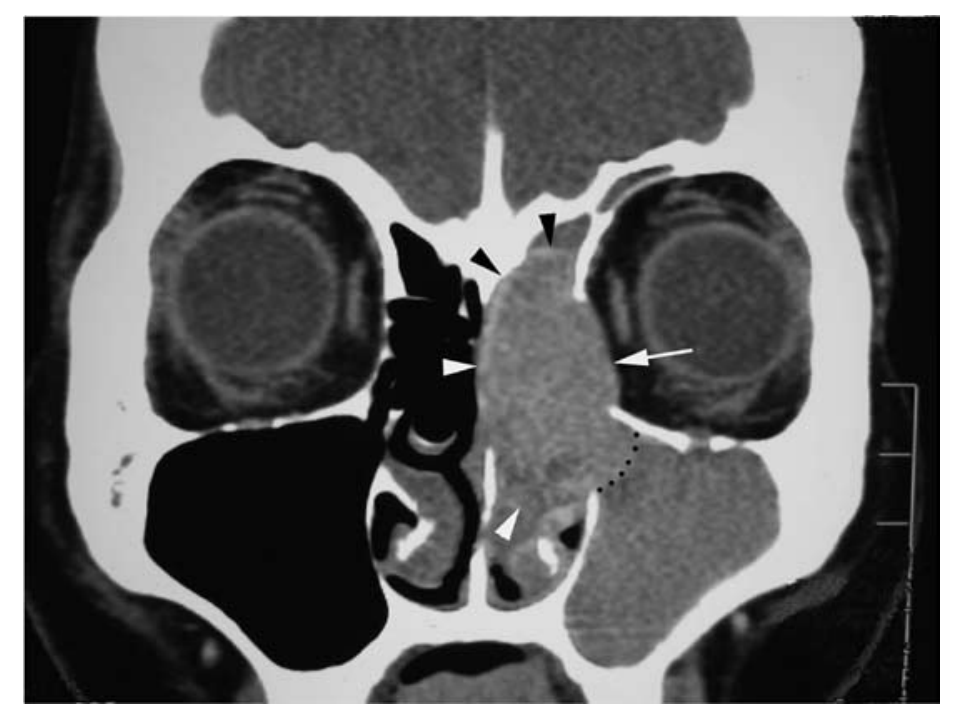

Figure 1. Contrast computed tomography scan, coronal section. A predominantly homogeneouslyenhancing mass (margins marked by arrowheads) is seen expanding the left nasal cavity, with minimal bulging of the nasal septum to the right and inferior compression of the ipsilateral inferior turbinate. The epicenter of the mass appears to be within the left anterior ethmoidal air cells. Thinning and displacement of ipsilateral lamina papyracea is noted (white arrow). No evidence of subperiosteal or extraconal extension is seen within the left orbit. Obliteration of the left maxillary ostium and infundibulum by the inferolateral aspect of the mass can be observed (black dotted line). Retained fluid within the left frontal \& maxillary sinuses from ostiomeatal complex obstruction is evident.

Ophthalmologic assessment was unremarkable. The patient underwent another CT scan to assess the extent of the residual tumor which showed a mass adhering to the periorbita, extending to the frontal ethmoidal recess. A repeat angiogram demonstrated that the feeding vessel of the tumor was the left ophthlamic artery and embolization was abandoned in view of the high risk of blindness. The patient subsequently underwent an endoscopic assisted medial maxillectomy via lateral rhinotomy. Histopathologic examination revealed nonkeratinizing (transitional) cell carcinoma of all tissue from the retrobullar area, frontal recess and cribiform plate.

Consequently the patient was advised to undergo intensity modulated radiotherapy in view of the residual tumor. Since the risk of complications was inevitable to such a vital sense organ, the patient declined. She is nevertheless under close regular follow-up.

\section{DISCUSSION}

Nonkeratinizing carcinoma (NKCa) is a rare malignancy of the nose and paranasal sinuses. The incidence of sinonasal malignancy is approximately 3.5 per 100,000 population per year. ${ }^{4}$ Of this, $15-20 \%$ are nonkeratinizing carcinoma. ${ }^{1}$ According to the WHO classification, it has many synonyms including Schneiderian carcinoma, transitional cell carcinoma, cylindrical cell carcinoma, Ringertz carcinoma and respiratory epithelial carcinoma. ${ }^{5}$

In the 1600s, Victor Conrad Schneider first described the mucosal 


\section{CASE REPORTS}

epithelium lining of the nasal cavity and the paranasal sinuses as ectodermal in origin. ${ }^{6}$ It is derived from nasal placodes that invaginate to form the primitive nasal sacs and ultimately the sinonasal cavities and lacrimal apparatus. The posterior boundary of this lining is the posterior choanae although it is continuous with the rest of the nasopharynx which is endodermally-derived i.e. from the foregut respiratory epithelium. Therefore, the use of the term Schneiderian distinguishes the boundaries of this epithelium and avoids confusion with any other anatomically-located tumors.?

The WHO classification also lists NKCa as a variant of squamous cell carcinoma. It is described as a tumor of the sinonasal tract characterized by a plexiform or ribbon-like growth pattern with occasional mucuscontaining cells. ${ }^{5}$ Although identified as nonkeratinizing, there are often small keratin pearls interspersed within the proliferations and some may form surface keratin that fills cystic spaces. ${ }^{5,8}$

In our patient, histopathological examination showed tissue partly lined by respiratory epithelium with the underlying stroma infiltrated by malignant cells forming islands and ribbon-like patterns. The cells displayed large nuclei with moderate pleomorphism, vesicular nuclei and prominent large nuclei. Some of the cells had 2 to 3 nucleoli. Mitoses were frequently seen. Bone trabeculae, areas of haemorrhage and necrosis were also present. No evidence of keratinisation was seen (Figures 2 and 3). Amelanotic mucosal malignant melanoma was ruled out by immunohistochemistry where there was negativity to Melan- $A$, HMB45 and S100.

The ribbon-like invasive architecture and monomorphic nuclear cytology of nonkeratinizing carcinoma may mimic inverted papilloma. Thus, Osborn called inverted papillomas as transitional papillomas and sinonasal nonkeratinizing carcinoma as transitional carcinomas. ${ }^{2}$ However, the focal keratin pearl formation, increased mitotic activity and nuclear pleomorphism distinguish the nonkeratinizing carcinoma. ${ }^{8}$ It may be impossible to identify NKCa from a carcinoma-ex-inverted papilloma characterized by diffuse dysplasia of the epithelium unless there is residual, better differentiated underlying inverted papilloma present. ${ }^{8}$ As reported by Robin et al. in 1979 and Svane-Knudsen et al. in 1998, a small percentage of transitional-type carcinomas may arise in pre-existing transitional cell papillomas. ${ }^{1,9}$ Most nonkeratinizing carcinomas are well-differentiated resembling transitional epithelium reminiscent of urothelium. Some are poorly-differentiated, composing layers of disordered small anaplastic cells though others show pseudostratified tall cylindrical cells with a basal palisade of columnar cells. ${ }^{8}$

The designation of cylindrical cell carcinoma as a synonym on the other hand is misleading as it may suggest a relationship to the

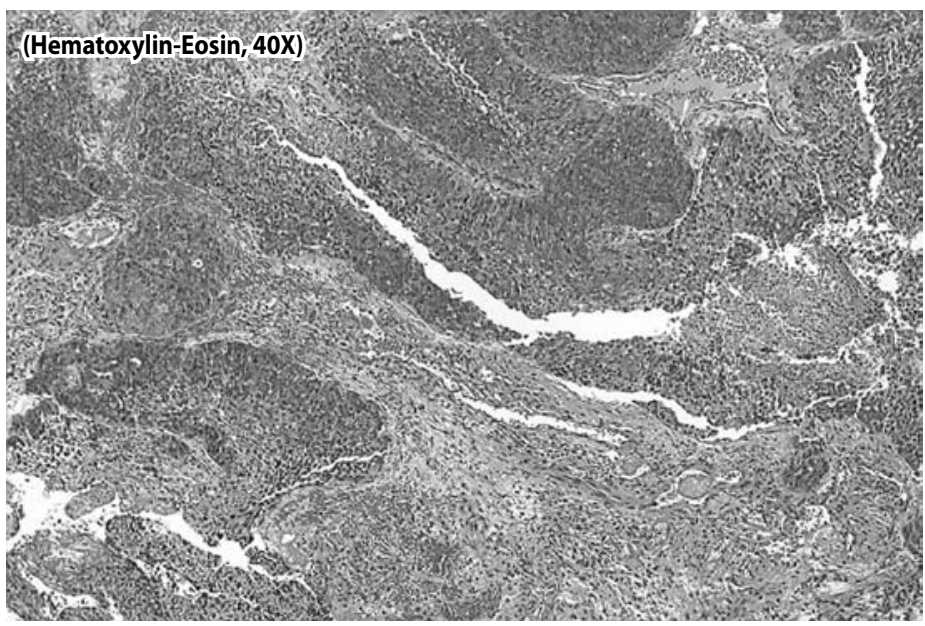

Figure 2. Histopathologic section. Hematoxylin and Eosin, low-power view (40X). Respiratory epithelium with the underlying stroma infiltrated by malignant cells forming islands and ribbon-like patterns.

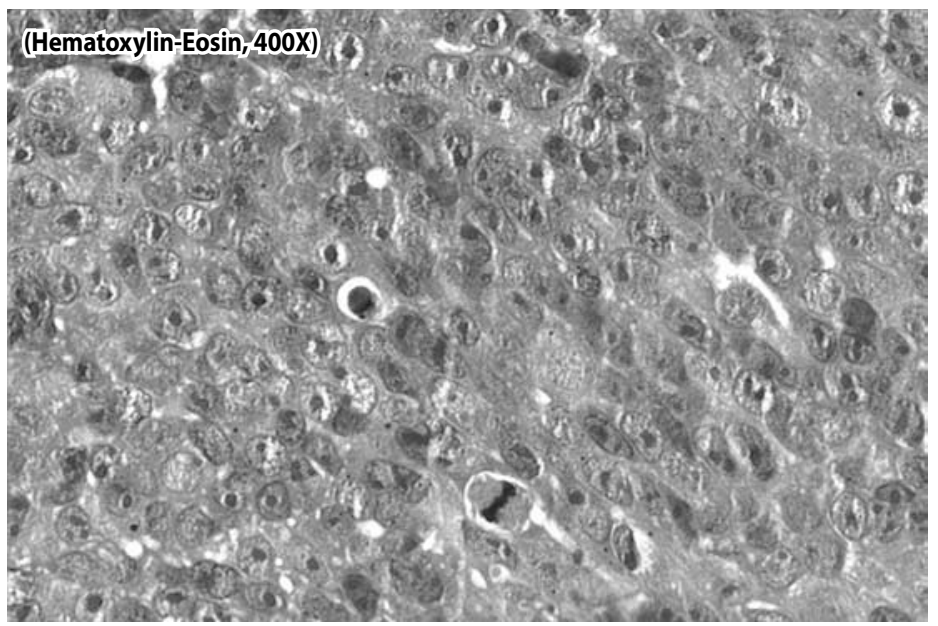

Figure 3. Histopathologic section. Hematoxylin and Eosin, high-power view (400X). The cells display large nuclei with moderate pleomorphism, vesicular nuclei and prominent large nuclei, some with 2 to 3 nucleoli and frequent mitoses. No evidence of keratinisation is seen.

cylindrical cell papilloma (oncocytic Schnederian papilloma). The latter is microscopically distinct characterized by surface oncocytic columnar and mucus cells and is unrelated to NKCa. ${ }^{3}$

The many different terminologies and synonyms that have been used frequently in the international literature may have lead to some confusion and perhaps misdocumentation of this rare tumor. In a series reported by Osborn in 1970 there were 57 cases of transitional cell carcinomas seen and treated in the Royal National Throat and Ear Hospital between 1948 and 1968 accounting for approximately $20 \%$ of all carcinomas of the nose and sinuses. ${ }^{2}$ A review by Robin et al. in 1979 illustrated a series obtained from registrations in the Birmingham Regional Cancer Registry from 1957 to 1972 inclusive of 
only 48 cases of transitional cell carcinomas constituting merely $7.7 \%$ of all malignant tumors of the nose and paranasal sinuses. ${ }^{1}$ Manivel et al. in 1986 reported two cases of transitional (cylindric) cell carcinoma with endodermal simus tumor-like features of the nasopharynx and paranasal sinuses. ${ }^{10}$ In 2000, Calderon-Garciduenas et al. published their series obtained from a major oncology hospital in metropolitan Mexico City from 1976-1997 which listed Schneiderian carcinoma as one of the diagnosis in their 256 patients. ${ }^{11}$ El-Mofty and Lu in 2005 reported only eight cases of nonkeratinizing carcinomas retrieved from the Department of Pathology and Immunology at Washington University School of Medicine, St. Louis Missouri, though they did not specify when they were diagnosed. ${ }^{3}$ Another series published in a Chinese journal reported one case of Schneiderian carcinoma from 39 ethmoidal malignancies. ${ }^{12} \mathrm{~A}$ recent case report evidently demonstrated incorrect terminology where the author used "sinonasal undifferentiated carcinoma" interchangeably with "schneiderian carcinoma."13 Some authors also regard NKCa as a distinct clinicopathologic entity. Justification for a separate classification is based on various significant observations. ${ }^{1,3}$ Robin et al. found a difference in the mean age of presentation between men and women in NKCa which was 57.8 years and 70.4 years, respectively. ${ }^{1}$ This difference was statistically significant and was greater than in other histological groups. They also found a marked contrast in the distribution of sites among the different types of carcinomas. Squamous cell carcinomas were seen predominantly in the maxillary antrum, adenocarcinomas were predominantly in the ethmoid while NKCas was more evenly spread. Another observation was that NKCa in men carried a better prognosis than in women where the five-year survival rates were $40 \%$ and $13 \%$ respectively. Other studies have found that it emerges more favorably with the five year survival rate of $37.5 \%$ compared to $\mathrm{KSCC}$ of $10 \%$ and is more sensitive to radiation but has a greater tendency to local recurrence. ${ }^{2,14} \mathrm{~A}$ recent study has also shown that NKCas of the sinonasal tract have a higher prevalence of high risk HPV DNA than other types of carcinomas in this region. ${ }^{3}$

NKCa is a rare malignancy of the nose and paranasal sinuses and should be recognized as a distinct clinicopathologic entity to determine the best treatment modality and to better predict the outcome of treatment. Nomenclature of this tumor should be standardized to avoid confusion and misdocumentation.

\section{REFERENCES}

1. Robin P, Powell DJ, Stansbie JM. Carcinoma of the nasal cavity and paranasal sinuses: incidence and presentation of different histologic types. Clin Otolaryngol Allied Sci. 1979 Dec; 4(6):431-56.

2. Osborn DA. Nature and behavior of transitional tumors of the upper respiratory tract. Cancer. 1970 Jan; 25(1):50-60.

3. El-Mofty S, Lu DW. Prevalence of high-risk human papillomavirus DNA in nonkeratinizing (cylindrical cell) carcinoma of the sinonasal tract. Am J Surg Pathol. 2005 Oct; 29(10):1367-72.

4. Muir CS, Nectoux J. Descriptive epidemiology of malignant neoplasms of nose, nasal cavities, middle ear and accessory sinuses. Clin Otolaryngol Allied Sci. 1980 Jun; 5(3):195-211.

5. Pilch BZ, Bouquot J, Thompson LDR. Squamous cell carcinoma. In: Barnes L, Eveson JW, Reichart P, Sidransky D, editors. World Health Organization classification of tumors. Pathology and genetics of head and neck tumors. Lyon: IARC Press; 2005.p.15-7.

6. Batsakis JG. Pathology Consultation. Nasal (Schneiderian) papillomas. Ann Otol Rhinol Laryngol. 1981 Mar-Apr; 90(2 Pt 1):190-1.

7. Batsakis JG, Suarez P. Schneiderian papillomas and carcinomas: A review. Adv Anat Pathol. 2001 Mar; 8(2):53-64.

8. Zarbo RJ, Torres FX, Gomez J. Nasal cavity and paranasal sinuses: embryology, anatomy, histology and pathology. In: Pilch BZ, editor. Head and Neck Surgical Pathology. Philadelphia: Lippincott Williams \& Wilkins; 2000.p.80-156.

9. Svane-Knudsen V, Jorgensen KE, Hansen O, Lindgren A, Marker P. Cancer of the nasal cavity and the paranasal sinuses: A series of 115 patients. Rhinology. 1998 Mar; 36(1):12-4.

10. Manivel C, Wick MR, Dehner LP. Transitional (cylindric) cell carcinoma with endodermal sinus tumor-like features of the nasopharynx and paranasal sinuses. Clinicopathologic and immunohistochemical study of two cases. Arch Pathol Lab Med. 1986 Mar; 110(3):198-202.

11. Calderon-Garciduenas L, Delgado R, Calderon-Garciduenas A, Meneses A, Ruiz LM, De La Garza $J$, et al. Malignant neoplasms of the nasal cavity and paranasal sinuses: a series of 256 patients in Mexico City and Monterrey. Is air pollution the missing link? Otolaryngol Head Neck Surg. 2000 Apr; 122(4):499-508.

12. Zhang TM, Fang JG, Chen XH, Zhang JL, Zhao JW. [Trans-cranio-naso-orbital approach in treatment of ethmoid sinus malignant tumors: analysis of 39 cases]. Zhonghua Yi Xue Za Zhi. 2007 Jan; 87(5):304-7.

13. Ahossi V, Vincent S, Duvillard C. Sinonasal undifferentiated carcinoma, or schneiderian carcinoma arising from an aspergillosis: a case history. Br J Oral Maxillofac Surg. 2009 Jun; 47(4):316-7.

14. Friedmann I, Osborn DA. Carcinoma of the surface epithelium. In: Freidmann I, editor. Pathology of granulomas and neoplasms of the nose and paranasal sinuses. Edinburgh: Churchill Livingstone; 1982.p.118-82. 